\title{
Diseño y aplicación de unidades didácticas y su aporte a la formación de futuros docentes de ciencias naturales de la Universidad Surcolombiana: resultados preliminares
}

Amórtegui Cedeño, Elías Francisco'

Categoría 2. Trabajo de investigación (en proceso)

\section{Resumen}

En este documento se presentan resultados preliminares acerca de la sistematización del diseño y aplicación de unidades didácticas para la enseñanza de las ciencias naturales. El grupo de trabajo consistió en 20 futuros docentes con edades entre los 20 y 25 años, pertenecientes a estratos socio-económicos 1 y 2 , que cursaron el espacio académico de Didáctica I durante el segundo semestre del 2013 en el Programa de Licenciatura en Ciencias Naturales: Física, Química, Biología de la Universidad Surcolombiana (Neiva, Colombia). La metodología estuvo enmarcada en un enfoque cualitativo, empleando el análisis de contenido y la sistematización de la primera y segunda entrega del diseño de las unidades didácticas. Los resultados muestran una tendencia hacia las concepciones constructivistas acerca de la enseñanza, los contenidos de enseñanza y los aspectos formativos docentes, en la segunda entrega, sin embargo para el caso de la primera, las concepciones suelen estar enmarcadas en modelos tradicionales.

\section{Palabras clave}

Unidades didácticas, Conocimiento Profesional, Conocimiento Didáctico, Enseñanza-aprendizaje.

\section{Objetivo}

Sistematizar el diseño y aplicación de unidades didácticas elaboradas por los estudiantes que cursan el espacio de Didáctica I del programa de Licenciatura en Ciencias Naturales: Física, Química, Biología, de la Universidad Surcolombiana.

\footnotetext{
${ }^{1}$ Universidad Surcolombiana. elias.amortegui@usco.edu.co
} 
Revista Tecné, Episteme y Didaxis: TED. Año 2014, Número Extraordinario. ISSN Impreso: 0121-3814, ISSN web: 2323-0126

Memorias, Sexto Congreso Internacional sobre Formación de Profesores de Ciencias. 08 al 10 de octubre de 2014, Bogotá

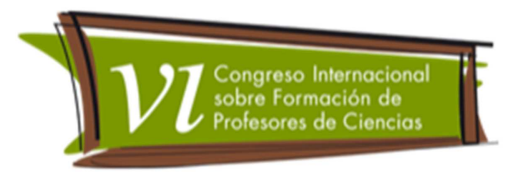

\section{Marco teórico}

El estudio está enmarcado en la perspectiva del Conocimiento Profesional del Profesor de Ciencias, la cual plantea que los profesores cuentan con un conocimiento particular que los distingue de los demás profesionales y son varios los autores que al respecto han hecho grandes a portes a la comprensión del tema (Shulman, 1986, 1987, Bromme, 1988, Tardif, 2004, Grossman, 1990, Marcelo, 1999, Valbuena, 2007, Amórtegui y Correa, 2012 y Amórtegui, 2011). Los estudios sobre el CPP se han centrado en dos grandes tendencias, que son: la caracterización y ubicación sobre el CPP y el reconocimiento del Conocimiento Didáctico del Contenido (CDC).

Para el caso de profesores de Ciencias Naturales en educación básica, los estudios desde la perspectiva del Conocimiento Profesional también son escasos tanto a nivel colombiano como internacional (Martínez y Molina, 2009). En términos globales Abell (2008) plantea que después de 20 años de investigación sobre el Conocimiento de los Profesores y particularmente sobre el Pedagogical Content Knowledge, aún queda una gran cantidad de investigaciones por realizar.

Así mismo, la revisión de antecedentes muestra que a nivel internacional existen algunos estudios que se han realizado con profesores de una disciplina, ya sea Biología, Química o Física, sin embargo las investigaciones con profesores de Ciencias Naturales y Educación Ambiental son escasas, resaltando así la importancia, relevancia y pertinencia de los presentes hallazgos preliminares (Amórtegui y Rivas, en prensa).

\section{Metodología}

El estudio se llevó a cabo desde un enfoque cualitativo, empleando el método de análisis de contenido en el proceso de sistematización. El grupo objeto de estudio consistió en 20 estudiantes con edades entre 20 y 25 años, pertenecientes a estratos socio-económicos 1 y 2 que cursaron el espacio académico de Didáctica I durante el segundo semestre del 2013, en el Programa de Licenciatura Ciencias Naturales: Física, Química, Biología de la Universidad Surcolombiana (Neiva, Colombia). El procedimiento para el análisis de contenido para esta investigación tuvo en cuenta los siguientes aspectos: primero se identificaron las fuentes de información, luego se ubicaron las unidades de información (UI) de cada fuente, las cuales corresponden a afirmaciones textuales en dichos 
Revista Tecné, Episteme y Didaxis: TED. Año 2014, Número Extraordinario. ISSN Impreso: 0121-3814, ISSN web: 2323-0126

Memorias, Sexto Congreso Internacional sobre Formación de Profesores de Ciencias. 08 al 10 de octubre de 2014, Bogotá

documentos que ofrecieron información con sentido y significado propio para la investigación, posteriormente se les asignó un código. Luego, las UI se sistematizaron de acuerdo a cuatro categorías que se encuentran enmarcadas dentro del Conocimiento Profesional del Profesor (Amórtegui y Correa, 2012): Enseñanza, Contenidos de enseñanza, Aprendizaje y Formación docente. Las unidades didácticas corresponden a tres grupos de trabajo, grupo 1 enseñanza de la genética, grupo 2 enseñanzas de la nutrición y grupo 3 enseñanzas de la respiración.

\section{Resultados}

A continuación se presentan los hallazgos en cada una de las categorías, en algunos casos se ilustra con declaraciones textuales de los futuros profesores de Ciencias Naturales (se destaca la información relevante subrayando).

\section{Contenidos de enseñanza}

En la primera entrega, la mayoría de docentes en formación establecieron como elementos en el diseño de la unidad didáctica aspectos relacionados exclusivamente desde la perspectiva tradicional de la enseñanza de las ciencias naturales en donde priman los contenidos conceptuales; tan sólo algunos estudiantes explicitaron la importancia de tener en cuenta elementos procedimentales y actitudinales.

G1.E1.3. "(Haciendo referencia a los objetivos de aprendizaje en la unidad didáctica) "Adquirir conceptos claros acerca de las bio-moléculas y los sistemas buffer como fuente de regulación de todo ser vivo; Entender el material genético como código universal de formación de seres vivos; Reconocer la composición química de los ácidos nucléicos $(A D N-A R N) . "$

Para el caso de la segunda entrega, hubo algunas modificaciones en los contenidos de enseñanza, ya que algunos de ellos tuvieron en cuenta elementos procedimentales y actitudinales, en parte, producto de las discusiones generadas a través del trabajo colaborativo del espacio académico de Didáctica I con base en las lecturas de Sánchez, De Pro bueno y Valcárcel (1997), De Pro bueno (1999), Sánchez y Valcárcel (1993) y Quintanilla, Merino y Daza (2010). De igual 
Revista Tecné, Episteme y Didaxis: TED. Año 2014, Número Extraordinario. ISSN Impreso: 0121-3814, ISSN web: 2323-0126

Memorias, Sexto Congreso Internacional sobre Formación de Profesores de Ciencias. 08 al 10 de octubre de 2014, Bogotá

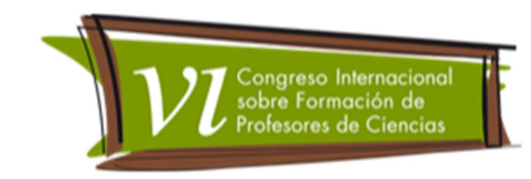

forma se realizaron análisis de videograbaciones de secuencias de clase de profesores en ejercicio y en formación inicial del Departamento del Huila.

G2.E2.10. (Haciendo referencia a los objetivos de aprendizaje en la unidad didáctica) "Desarrollar un pensamiento reflexivo acerca de los hábitos alimenticios que se mantienen en nuestro país y en la región; Modificar la dieta alimenticia, haciéndola verdaderamente provechosa para nuestro organismo; Fomentar un espiritu investigativo-práctico que permita mejorar el nivel de aprendizaje."

Lo anterior, está relacionado en su mayoría con la perspectiva academicista de la enseñanza de las ciencias, en la cual priman los contenidos conceptuales y por otro lado en menor proporción una perspectiva significativa-sistémica en la cual se propende por la interrelación de manera integral de los contenidos conceptuales, procedimentales y actitudinales (Valbuena, 2007). Esto, con el propósito de atribuir sentido al mundo de lo vivo y de su entorno, y que, además, posibiliten aplicar dichos conocimientos de una manera significativa y crítica a su vida cotidiana mediante la valoración, conservación y aprovechamiento sostenible de los recursos, más aún cuando en la ciudad de Neiva existen problemáticas ambientales, tales como: el abandono y la falta de conciencia de la comunidad en el manejo de los recursos hídricos como es el caso de la Laguna del Curibano, el mal manejo de los desechos sólidos, la tala de árboles, la poca arborización, la contaminación auditiva, entre otros.

Por otra parte, la situación anterior está relacionada con la estructura curricular del programa, ya que la formación en Didáctica de las Ciencias Naturales corresponde exclusivamente al sexto y séptimo semestre y, además, a que las prácticas pedagógicas se llevan a cabo únicamente durante el último año de formación.

\section{Enseñanza}

Con relación a las ideas sobre enseñanza, se destacaron en ambas entregas los elementos relacionados con enfoques constructivistas en los que es fundamental tener en cuenta el interés y curiosidad de los estudiantes; algunos de los futuros maestros consideran importante que la enseñanza de estas disciplinas pueda generar sentido investigativo en los estudiantes dentro y fuera del aula. 
Revista Tecné, Episteme y Didaxis: TED. Año 2014, Número Extraordinario. ISSN Impreso: 0121-3814, ISSN web: 2323-0126

Memorias, Sexto Congreso Internacional sobre Formación de Profesores de Ciencias. 08 al 10 de octubre de 2014, Bogotá

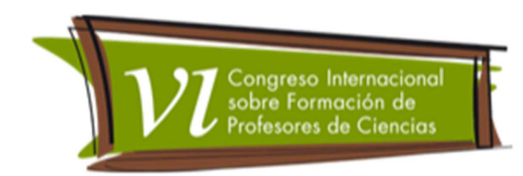

G3.E1.5 (Haciendo referencia a los aspectos de la enseñanza en la unidad didáctica) "incorporar un dinamismo constructivo dentro y fuera del aula de clase, generando en los alumnos interés, curiosidad y a su vez cultivando el sentido de investigación. "

Es fundamental entonces que los futuros maestros de Ciencias Naturales reconozcan que las estrategias y actividades de enseñanza que se implementan, en este caso las planteadas en la unidad didáctica, además de tener en cuenta las concepciones de los alumnos, deben partir de su nivel de desarrollo cognitivo, sus intereses, sus experiencias y su contexto sociocultural (Amórtegui, 2011), tal como lo muestra la siguiente afirmación, en la que se hace explícita la relación entre las características de la alimentación en el contexto cotidiano colombiano.

G2.E2.10 (Haciendo referencia a la justificación de la Unidad Didáctica) "Por medio de la práctica y asimilación de lo cotidiano, tomando como eje central la tradición alimenticia Colombiana y del mundo, que mantiene hábitos de alimentación basados en paradigmas del sistema económico imperante del mundo, y no basados en la supervivencia como tal."

\section{Aprendizaje}

Cabe destacar que tan sólo uno de los grupos identificó elementos relacionados con las características sociales de los estudiantes y su influencia en el aprendizaje de los contenidos científicos en la escuela, principalmente debido a que tanto profesores en formación como estudiantes han vivido en dichas características, que para el caso del Municipio de Neiva son compartidas por el pequeño número de instituciones educativas oficiales en comparación de ciudades de mayor área geográfica (Neiva cuenta con alrededor de 300.000 habitantes); lo anterior está relacionado con que las primeras ideas que tienen los docentes en sus primeros años de formación, con relación al ámbito educativo, provienen en gran parte de su experiencia como aprendices en la escuela primaria, media y secundaria (Amórtegui y Correa, 2012). 
Revista Tecné, Episteme y Didaxis: TED. Año 2014, Número Extraordinario. ISSN Impreso: 0121-3814, ISSN web: 2323-0126

Memorias, Sexto Congreso Internacional sobre Formación de Profesores de Ciencias. 08 al 10 de octubre de 2014, Bogotá

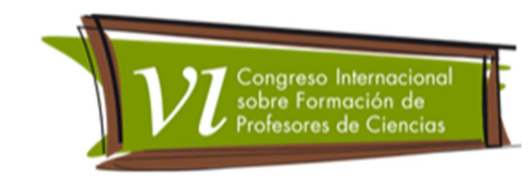

G3.E2.8 (Haciendo referencia al contexto de los estudiantes de la Unidad Didáctica) "algunos de estos estudiantes vienen de familias con problemas intrafamiliares, escasos recursos económicos y pocas posibilidades para la utilización y aprovechamiento del tiempo libre y la recreación, es por esto que se hace necesario crear escenarios que potencien estas falencias y se aproveche al máximo el potencial humano desde la escuela."

\section{Aspectos formativos docentes}

En algunos de los casos, los docentes en formación se referían al aporte de la unidad didáctica en su primera entrega tan sólo en términos Instrumentalistas, en la cual lo importante de esta actividad se reducía a la aplicación de actividades de enseñanza sin tener en cuenta ningún elemento de transformación didáctica, identidad profesional o construcción de conocimiento didáctico del contenido.

\section{G1.E1.7 (Haciendo referencia a los objetivos formativos de la unidad didáctica) "Aplicar estrategias de enseñanza y aprendizaje."}

Para el caso de la segunda entrega, la mayoría de estudiantes tuvieron en cuenta elementos de transformación didáctica y el aporte de la unidad didáctica a la profesionalización de la docencia, a partir de los procesos de reflexión y la formación ética-moral de los maestros frente a sus estudiantes.

G3.E2.1 (Haciendo referencia a los objetivos formativos de la unidad didáctica) "Asegurar la adquisición de las competencias profesionales definidas como necesarias para educar en contextos educativos heterogéneos...Garantizar los conocimientos necesarios y herramientas didácticas en un enfoque interdisciplinar y transdisciplinar ante la necesidad de formarse y de reflexionar a lo largo de la vida profesional, promoviendo para ello experiencias educativas de innovación docente que la favorezcan." 
Revista Tecné, Episteme y Didaxis: TED. Año 2014, Número Extraordinario. ISSN Impreso: 0121-3814, ISSN web: 2323-0126 Memorias, Sexto Congreso Internacional sobre Formación de Profesores de Ciencias. 08 al 10 de octubre de 2014, Bogotá

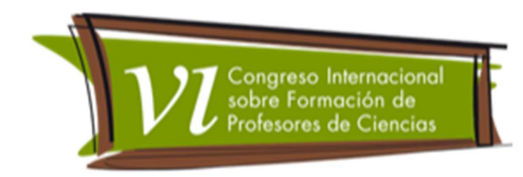

En primera medida, cabe resaltar que estas afirmaciones de los futuros maestros trascienden la idea predominante en la cual basta con que el profesor domine académicamente el conocimiento disciplinar que enseña, para que sea un buen profesor. Sin embargo, hay que destacar que la mayoría de profesores en formación conciben la unidad didáctica exclusivamente desde una perspectiva instrumental o activista, en la que se enfocan las actividades y las formas de enseñar contenidos biológicos. Lo ideal sería lograr que los futuros docentes de Ciencias Naturales conciban la unidad didáctica como un ejercicio formativo que les permita la integración de contenidos escolares, estrategias metodológicas, finalidades de enseñanza, ideas de los alumnos sobre las ciencias naturales, dificultades de enseñanza-aprendizaje y evaluación de los aprendizajes, en aras de que reconozcan que el diseño de la unidad didáctica permite identificar el conocimiento que se construye en el aula de clase y que este difiere del conocimiento producido por los científicos, pues se trata de un conocimiento escolar epistemológicamente diferenciado, que ha de producirse didácticamente a partir de las transformaciones e integraciones de diferentes saberes y conocimientos que confluyen en la escuela (Valbuena et al, 2009).

Teniendo en cuenta lo anterior, es importante posibilitar que en la formación de maestros se trabaje porque éstos se reconozcan como sujetos de conocimientos propios de su profesión. La formación del profesorado debe partir de un enfoque reflexivo, en el que se analicen las prácticas profesionales, las tareas y los conocimientos de los maestros (Tardif, 2004). Barnett y Hodson (2001) consideran que no basta con que el docente adquiera competencias para aplicar los referentes teóricos sino requiere, además, poseer un conocimiento tal que lo capacite para tomar decisiones en contextos educativos particulares.

\section{Conclusiones}

Con relación a la enseñanza, los docentes en formación conciben la importancia del uso de las ideas previas, el interés, la curiosidad y la necesidad de romper con la educación tradicional; frente a los contenidos de enseñanza, los estudiantes establecieron como elementos en el diseño de la unidad didáctica aspectos relacionados exclusivamente desde la perspectiva tradicional de la enseñanza de las Ciencias Naturales en donde priman los contenidos conceptuales; sin embargo, algunos estudiantes explicitan la importancia de tener en cuenta elementos procedimentales y actitudinales; con relación al aprendizaje tan sólo un grupo dio cuenta de la importancia del contexto de los estudiantes como un elemento que incide en el aprendizaje de las Ciencias Naturales y en cuanto a 
Revista Tecné, Episteme y Didaxis: TED. Año 2014, Número Extraordinario. ISSN Impreso: 0121-3814, ISSN web: 2323-0126

Memorias, Sexto Congreso Internacional sobre Formación de Profesores de Ciencias. 08 al 10 de octubre de 2014, Bogotá

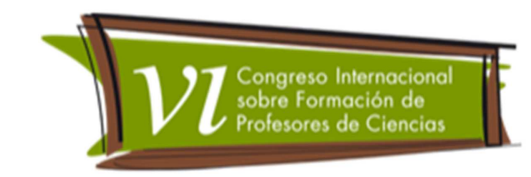

los aspectos formativos, establecen la importancia de la profesionalización docente, la identidad y la reflexión.

\section{Referencias bibliográficas}

Abell, S. (2008). Twenty Yaers Later: Does Pedagogical Content Knowledge remain a useful idea? International Journal of Science Education. 30 (10), 1405-1416.

Amórtegui, E (2011). Concepciones sobre prácticas de campo y su relación con el conocimiento profesional del profesor, de futuros docentes de Biología de la Universidad Pedagógica Nacional. Título para optar al título de Magíster en Educación de la Universidad Pedagógica Nacional. Departamento de Posgrados: Bogotá.

Amórtegui y Correa (2012). Planificación de las prácticas de campo en el proyecto curricular de licenciatura en biología de la Universidad Pedagógica Nacional. Caracterización desde la perspectiva del Conocimiento Profesional del Profesor de Biología. Bogotá: Fundación Franciska Radke.

Amórtegui, E \& Rivas, J (en prensa). Aporte del diseño de unidades didácticas a la formación de futuros docentes de ciencias naturales y educación ambiental de la Universidad Surcolombiana. Revista Bio-Grafia. Escritos sobre Biología y su enseñanza.

Barnett, J. y D. Hodson (2001), "Pedagogical context knowledge: toward a fuller understanding of what good science- teachers know", Science Teacher Education, núm. 85, pág. 426-453.

Bromme, R. (1988). Conocimientos profesionales de los profesores. Enseñanza de las Ciencias, 6 (1), 19-29.

De Pro bueno, A (1999). Planificación de unidades didácticas por los profesores: análisis de tipos de actividades de enseñanza. Enseñanza de las ciencias. 17 (3), pág. 411-429

Grossman, P. (1990). The Making of a Teacher. Theacher Knowledge and Teacher Education. New York: Teachers College, Columbia University. 
Revista Tecné, Episteme y Didaxis: TED. Año 2014, Número Extraordinario. ISSN Impreso: 0121-3814, ISSN web: 2323-0126

Memorias, Sexto Congreso Internacional sobre Formación de Profesores de Ciencias. 08 al 10 de octubre de 2014, Bogotá

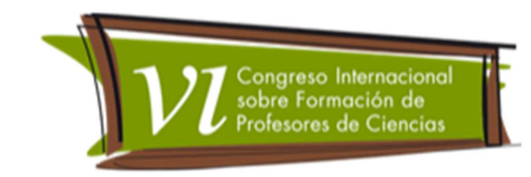

Jiménez, M (2000). Modelos didácticos. En: Perales J y Cañal P (Coord). Didáctica de las Ciencias Experimentales. Editorial Marfil Alcoy: España.

Marcelo, C. (1999). Cómo conocen los profesores la materia que enseñan. Algunas contribuciones de la investigación sobre conocimiento didáctico del contenido. En: MONTERO, L. y VEZ, J. (Eds.). Las didácticas específicas en la formación del profesorado. Santiago de Compostela: Tórculo. pág. 151185.

Martínez, c \& Molina, A (2009). El conocimiento profesional de los profesores de ciencias de primaria sobre el conocimiento escolar en el distrito capital: un problema de investigación. Memorias I congreso Nacional de investigación en educación en ciencias y tecnología, 2009, Junio 22 a 26.

Pozo, J y Gómez, M (1998). Aprender y enseñar ciencia. Del conocimiento cotidiano al conocimiento científico. Madrid: Ediciones Morata.

Quintanilla, M; Merino, C; Daza, S (2010). Unidades didácticas en química. Su contribución a la promoción de competencias de pensamiento científico. Barrancabermeja: Diseños litodigital.

Sánchez, B \& Valcárcel, P (1993). Diseño de unidades didácticas en el área de ciencias experimentales. Enseñanza de las ciencias. 11 (I), pág. 33-44.

Sánchez, B De Pro bueno, A, Valcárcel, P (1997). La utilización de un modelo de planificación e unidades didácticas: el estudio de las disoluciones en la educación secundaria. Enseñanza de las ciencias. 15 (I), pág. 35-50

Shulman, L. (1986). Those who understand: Knowledge growth in teaching. Educational Researcher, 15 (2), 4-14.

Shulman, L (1987). Knowledge and teaching. Foundations of the new reform. Harvard Educational Review. 57 (1).

Tardif, M. (2004). Los saberes del docente y su desarrollo profesional. Traducción de Pablo Manzano. Madrid: Narcea.

Valbuena, E. (2007). El Conocimiento Didáctico del Contenido Biológico. Estudio de las concepciones disciplinares y didácticas de futuros docentes de la 
Revista Tecné, Episteme y Didaxis: TED. Año 2014, Número Extraordinario. ISSN Impreso: 0121-3814, ISSN web: 2323-0126

Memorias, Sexto Congreso Internacional sobre Formación de Profesores de Ciencias. 08 al 10 de octubre de 2014, Bogotá

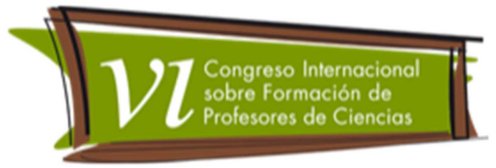

Universidad Pedagógica Nacional (Colombia). Tesis para optar al título de Doctor en Didáctica de las Ciencias Experimentales. Universidad Complutense de Madrid.

Valbuena, Édgar; Gutiérrez, Alice; Correa, Mónica; y Amórtegui, Elías. (2009). Procesos formativos que favorecen la construcción del conocimiento profesional del profesor en futuros docentes de Biología. Revista Colombiana de Educación. No 56. Pág. 156-179. 